\title{
Methanol adsorption by amorphous silica alumina in the critical temperature range
}

\author{
M. Kuczynski, A. van Ooteghem, and K. R. Westerterp \\ Chemical Reaction Engineering Laboratories, Department of Chemical Engineering, Twente University of Technology, Enschede, The \\ Netherlands
}

\begin{abstract}
The methanol adsorption capacity of an amorphous silica-alumina was measured using an equilibrium technique. The experimental temperature range was of 140 to $260^{\circ} \mathrm{C}$ and the pure methanol pressure range was 0.1 to $1.2 \mathrm{MPa}$. A multilayer adsorption was found, also for temperatures above the critical temperature of the adsorbate. Based on the Jovanovic adsorption model, the mean residence times of the adsorbed molecules were calculated. Surprisingly, the heat of adsorption was found to be independent of the temperature in the multilayer adsorption range.
\end{abstract}

Key words: Methanol [67-56-1], high temperature adsorption, silica-alumina, multilayers, adsorption heat.

\section{Notation}

Symbols

$a \quad=$ parameter defined by Equation (7)

$a^{\prime}=$ parameter defined by Equation (10)

$a(T)=$ parameter in the Peng-Robinson equation $\mathrm{Pa} /\left(\mathrm{mol} / \mathrm{m}^{3}\right)^{2}$

$b \quad=$ parameter defined by Equation (8)

$b^{\prime} \quad=$ parameter defined by Equation (11)

$b(T)=$ parameter in the Peng-Robinson equation $\mathrm{m}^{3} \mathrm{~mol}^{-1}$

$c=$ constant in the BET equation

$k=$ Boltzmann constant, being $1.3806 \cdot 10^{-23} \mathrm{JK}^{-1}$

$K=$ characteristic constant in the PengRobinson equation

$m$ = mass of one adsorbate molecule

$p \quad=$ pressure

$q \quad=$ adsorption capacity

$Q \quad=$ heat of adsorption

$R$ = gas constant, being 8.314

$T=$ absolute temperature

$V=$ molar volume

$x \quad=$ relative pressure $\left(=p / p_{o}\right)$

$\sigma \quad=$ active molecule area

$=$ residence time

$=$ acentric factor
Units

$-$

$-$

$\mathrm{Pa}^{-1}$

$-$

$\mathrm{kg}$

$\mathrm{Pa}$

$\mathrm{g} / \mathrm{g}$

$\mathrm{J}^{\mathrm{mol}}{ }^{-1}$

$\mathrm{J} \mathrm{mol}^{-1} \mathrm{~K}^{-1}$

$\mathrm{K}$

$\mathrm{m}^{3} \mathrm{~mol}^{-1}$

$-$

$\mathrm{m}^{2}$

$s$

$-$

\section{Introduction}

A theoretical interpretation of the experimental equilibrium data for adsorption is still difficult, particu-

larly at high absorbate pressures and temperatures. Generally, the reliability of the existent theoretical expressions for adsorption equilibria decreases rapidly with increasing pressure. In this paper, we present the results of our adsorption measurements for methanol on the low alumina, LAC-25 cracking catalyst of AKZO Amsterdam. We consider the multilayer adsorption theory of Jovanovic to be valid also above the critical temperature of the adsorbate. Therefore, we used his adsorption isotherm expressions to fit our experimental data.

\section{Experimental}

The experimental system is schematically shown in Figure 1. Prior to each experiment, the solid sample was dried for several hours at $280^{\circ} \mathrm{C}$ under vacuum, weighted and placed in a $570 \cdot 10^{-6}$ $\mathrm{m}^{3}$ autoclave. Subsequently, the air was evacuated from the autoclave at $280^{\circ} \mathrm{C}$ and the apparatus was cooled to room temperature. A precisely measured volume of liquid methanol was then sucked into the autoclave. The valves $V_{1}$ and $V_{2}$ are used to evacuate the air and to inject the methanol samples, respectively. After evacuation and injection, plugs were installed on the valves in order to prevent leaking. The pressure in the autoclave was measured at several temperatures between 140 and $260^{\circ} \mathrm{C}$, each time after thermal stabilization of the system. An electronic pressure transducer was used for 


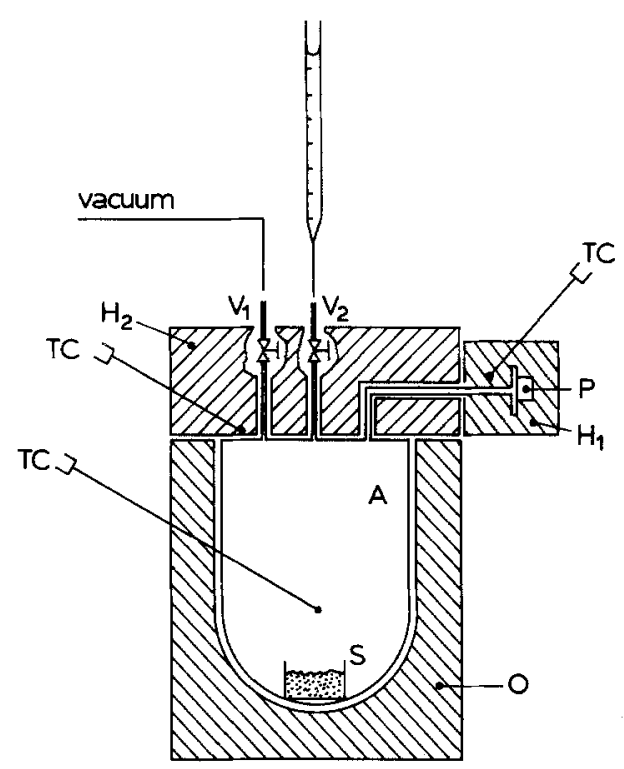

Fig. 1. The experimental set-up. Significance of symbols: $A=570$. $10^{-6} \mathrm{~m}^{3}$ stainless steel autoclave; $V_{1}=$ valve for evacuation; $V_{2}=$ methanol injection valve; $O=$ autoclave oven; $H_{1}, H_{2}=$ heating elements; $T C=$ thermocouples; $P$ electronic pressure transducer; $S=$ sample

the pressure measurements. The temperature of the transducer was kept constant at $200 \pm 0.1^{\circ} \mathrm{C}$, in order to prevent thermal drift of its calibration line. This procedure was repeated for various amounts of methanol injected, from 1 to $7 \cdot 10^{-6} \mathrm{~m}^{3}$. The weight of the solid sample was $8.75 \mathrm{~g}$. After the experiments, the composition of the gaseous autoclave contents was determined by means of gas chromatographic and NMR measurements. No decomposition products of methanol could be detected although dimethyl ether, for example, might be expected at the higher temperature levels investigated. There was no adsorption hysteresis observed, either. The same experimental procedure, but without the adsorbent, was used to obtain the most appropriate equation of state for methanol in the pressure and temperature range of our adsorption measurements.

\section{Results}

The amount of methanol adsorbed was calculated from the difference between the methanol pressure measured without and with the solid sample in the autoclave. Our experimental data obtained without the adsorbent were compared to the predictions of various equations of state for methanol. The experimental data agree well with the two-constant PengRobinson equation of state [1]:

$$
p=\frac{R T}{V-b}-\frac{a(T)}{V(V+b)+b(V-b)}
$$

in which

$$
a(T)=0.45724 \frac{R^{2} T_{c}^{2}}{P_{c}}\left(1+K\left(1-T_{r}\right)\right)^{2}, \mathrm{Nm}^{4} \mathrm{~mol}^{-2}
$$

$$
K=0.37464+1.54226 \omega-0.26992 \omega^{2}
$$

and

$$
b(T)=b\left(T_{c}\right)=0.07780 \frac{R T_{c}}{P_{c}}, \mathrm{~m}^{3} \mathrm{~mol}^{-1}
$$

where $\omega$ is the acentric factor for methanol. In Figure 2 our experimental data are compared with the predictions of the Peng-Robinson and the Beattie-Bridgeman [2] equations of state. On the vertical axis the procentual differences in the predictions of pressure are plotted, with respect to the measured ones. Other equations of state, e. g. the virial equation, gave differences of more than $2 \%$ with respect to our results. Equation (1) gives also a good fit of the $p-V-T$ data for methanol found in Literature $[2,3]$, and therefore we used it in the calculations.

Based on the most recent vapour-liquid equilibrium data for methanol by Ambrose et al. [4] we recalculated a value of 0.564 for the acentric factor of methanol, with the relation:

$$
\omega=-\log _{10}\left(P_{r}^{\mathrm{sat}}\right)_{T_{r}=0.7}-1 .
$$

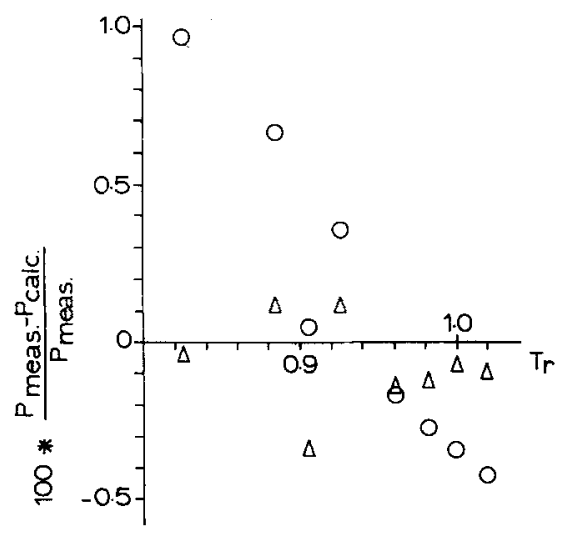

Fig. 2. Comparison of the experimental $p-V-T$ data with the predicted values with the Peng-Robinson $(\Delta)$ and Beattie-Bridgeman (O) equations of state. $5.5 \cdot 10^{-6} \mathrm{~m}^{3}$ methanol injected into the 570 . $10^{-6} \mathrm{~m}^{3}$ autoclave 
Table 1. Surface area distribution of the low alumina cracking catalysta ${ }^{2}$ )

\begin{tabular}{lc}
\hline $\begin{array}{l}\text { Pore diameter range } \\
\mathrm{nm}\end{array}$ & $\begin{array}{l}\text { Surface area } \\
\mathrm{m}^{2} / \text { gram }\end{array}$ \\
\hline $2.0-3.0$ & 92.6 \\
$3.0-4.0$ & 101 \\
$4.0-5.0$ & 138.1 \\
$5.0-6.0$ & 89.2 \\
$6.0-7.0$ & 38.4 \\
$7.0-9.0$ & 31 \\
$9.0-13.0$ & 10 \\
\hline
\end{tabular}

${ }^{2}$ ) These results were obtained in a BET apparatus. It was assumed that all pores have a cylindrical shape.

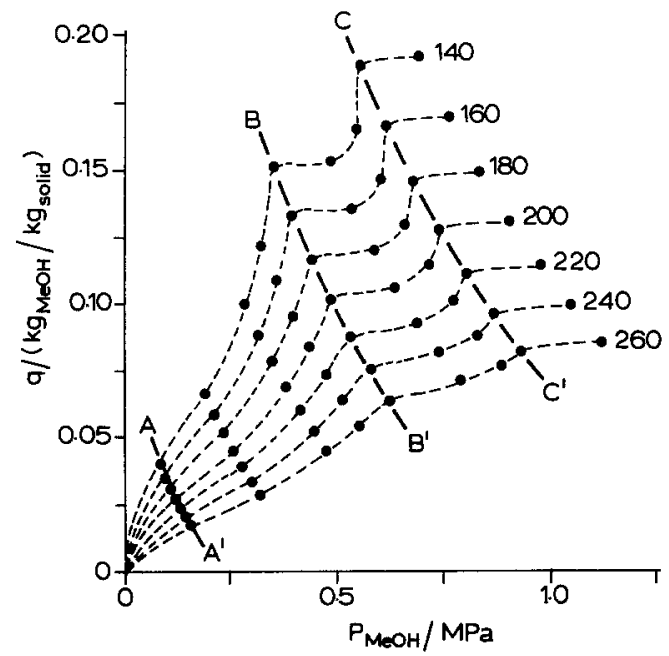

Fig. 3. The adsorption isotherms for methanol on the silica-alumina cracking catalyst. Parameter is the temperature in ${ }^{\circ} \mathrm{C}$

The pore diameter distribution of the adsorbent was determined using the usual BET technique; the results are given in Table 1. The measured adsorption isotherms are shown in Figure 3.

\section{Discussion and interpretation}

It is obvious that the complicated profile of our isotherms cannot be described by any of the usual adsorption isotherm expressions, such as the BET isotherm or its modifications, for example.

A surface area determination by the BET method gave the value of $505 \pm 9 \mathrm{~m}^{2} / \mathrm{g}$ solid. Taking the value of $0.212 \mathrm{~nm}^{2}$ for the effective area of one adsorbed methanol molecule [5], the maximum possible monolayer capacity is $0.127 \mathrm{~g} \mathrm{MeOH} / \mathrm{g}$ solid, while our experimental results for the adsorption capacity go up to
$0.192 \pm 0.006 \mathrm{~g} / \mathrm{g}$. This indicates a multilayer adsorption. Another feature of our adsorption isotherms supporting the multilayer adsorption hypothesis is the convexity of the isotherms toward the pressure axis in the range between the lines $A A^{\prime}$ and $B B^{\prime}$ in Figure 3 and the apparent existence of an inflexion point in the left part of this area. Such $S$-shaped isotherms are commonly considered to correspond with multilayer adsorption.

This inference is in disagreement with the essential assumption of the multilayer adsorption theories as given by Hüttig [6], Brunauer, Emmett and Teller [7] and modifications of the BET theory [8-12]. According to these authors multilayer formation should be impossible in our pressure and temperature range, which is above the boiling point of methanol.

As is well known the BET equation given by Equation (5)

$$
\frac{q}{q_{m}}=\frac{c x}{(1-x)(1-x+c x)}
$$

is quite inaccurate for relative pressures higher than 0.6 or 0.7 . Usually $c \gg 1$ [7], further the term $(1-x)$ in the denominator of the BET expression becomes significant at high pressures making the isotherm convex towards the pressure axis. Although $(1-x)$ gives a correct "boundary" behaviour of the isotherms at pressures tending to the saturation pressure of the adsorbate, the quantitative description of the multilayer adsorption is still inaccurate if $\frac{1}{(1-x)}$ becomes significant. Generally, the modifications of the BET theory all introduce correction terms to the heat of liquefaction for the subsequent adsorbed layers, still using the saturation pressure as a temperature dependent parameter. In this manner, the saturated vapor pressure is being ascribed to a "liquid-like" adsorbed phase consisting of a number of adsorbed layers having different binding energies. This approach is rather dubious in our opinion.

In 1969 Jovanovic [13] proposed a new adsorption theory for a homogeneous surface without lateral interactions between the adsorbed molecules. The Jovanovic isotherm is expressed by the following equation:

$$
q=q_{m}\{1-\exp (-a x)\} \exp (b x)
$$

where $q$ is the adsorbed amount, $q_{m}$ is the monolayer adsorption capacity, and $x$ is the relative pressure, defined as the ratio of the adsorbate pressure to its satu- 
ration pressure. The term $1-\exp (-a x)$ represents the monolayer adsorption, and the term $\exp (b x)$ is the correction for the multilayer adsorption. The parameters $a$ and $b$ describe the monolayer and multilayer adsorption, respectively:

$$
\begin{aligned}
& a=\sigma \tau p_{o} / \sqrt{2 \pi m k T} \\
& b=\sigma \tau_{L} p_{o} / \sqrt{2 \pi m k T}
\end{aligned}
$$

where $\sigma$ is the active area of one adsorbed molecule, $p_{o}$ is the saturation pressure, and $\tau$ and $\tau_{L}$ are the mean residence times in the adsorbed state in the first and in the higher layers, respectively.

Jovanovic assumed for the case of a multilayer adsorption that the molecules adsorbed in the higher layers do not deprive the molecules in the underlying layers of their capability for desorption. The adsorbed phase is thus considered as a zone of compressed gas rather than a liquid-like phase. Therfore, the phenomenon of multilayer adsorption has no relation at all to the saturation pressure and Equations (6)-(8) have to be rewritten for multilayer adsorption as follows:

$$
q=q_{m}\left\{1-\exp \left(1-a^{\prime} p\right)\right\} \exp \left(b^{\prime} p\right)
$$

with

$$
\begin{aligned}
& a^{\prime}=\sigma \tau / \sqrt{2 \pi m k T} \\
& b^{\prime}=\sigma \tau_{L} / \sqrt{2 \pi m k T} .
\end{aligned}
$$

Equations (9)-(11) are essentially the original expressions obtained by Jovanovic. He used the relative pressure $x$ in Equations (6)-(8), because, as he writes, in some cases it is more convenient to use relative instead of absolute pressures. In our opinion, the only advantage of Equations $(6)-(8)$ is that they can be easily used for the elaboration of the literature data, as was done by Jovanovic in his subsequent paper [14]. A number of examples of the use of the Jovanovic equations in a dimensionless form can also be found in the Literature [15-17]. We believe that the equations of Jovanovic are also valid above the critical temperature of the adsorbate and therefore that the introduction of the saturation pressure is inappropriate.

If we only consider the lines to the left of the line $B-$ $B^{\prime}$ in Figure 3, our experimental isotherms can be treated as type $\Pi$ isotherms according to Jovanovic's classification. Jovanovic $[13,14]$ and Yaronets and Yaronets [15] showed that the monolayer capacity $q_{m}$ and the multilayer adsorption parameter $b$ can be obtained from the convex segment of the adsorption isotherm, because in a semi-logarithmic plot of $q$ vs. $x$ a straight line will be obtained with $q_{m}$ as the intercept of the $y$ axis and with $b$ as the slope. In our case the experimental points in the region between the lines $A A^{\prime}$ and $B B^{\prime}$ in Figure 3 form straight lines in the semi-logarithmic $q$ vs. $p$ plot as shown in Figure 4. The values of $q_{m}$ and $b^{\prime}$ were calculated by linear regression; the results are presented in Table 2 . It can be seen that the monolayer capacity $q_{m}$ decreases with increasing temperature. Our experiments did not cover the low pressure range where the adsorption isotherms are expected to be concave toward the pressure axis. Therefore, we are not able to determine experimentally the monolayer adsorption parameter $a^{\prime}$.

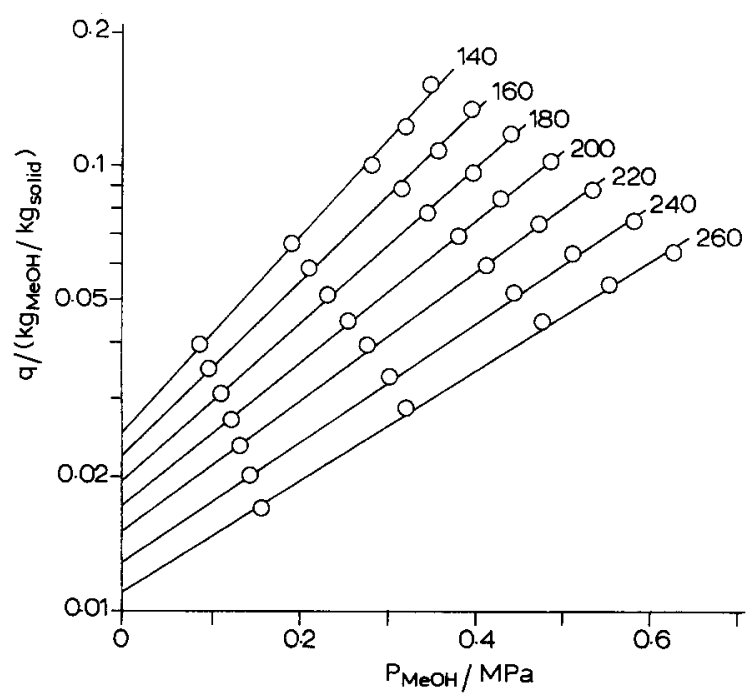

Fig. 4. The experimental adsorption capacity as a function of the methanol pressure. Parameter is the temperature of the system in ${ }^{\circ} \mathrm{C}$

Table 2. The values of $q_{m}$ and $b^{\prime}$

\begin{tabular}{lll}
\hline$T$ & $q_{m}$ & $b^{\prime} \cdot 10^{6}$ \\
$\mathrm{~K}$ & $\mathrm{~Pa}_{\mathrm{MeOH}} / \mathrm{g}_{\text {solid }}$ & \\
\hline 413 & 0.0256 & 4.92 \\
433 & 0.0226 & 4.39 \\
453 & 0.0199 & 3.95 \\
473 & 0.0175 & 3.59 \\
493 & 0.0152 & 3.30 \\
513 & 0.0131 & 3.07 \\
533 & 0.0112 & 2.83 \\
\hline
\end{tabular}


Table 3. The mean residence times of the adsorbed molecules

\begin{tabular}{ll}
\hline$T$ & $\tau_{L} \cdot 10^{10}$ \\
$\mathrm{~K}$ & $s$ \\
\hline 413 & 5.06 \\
433 & 4.63 \\
453 & 4.26 \\
473 & 3.96 \\
493 & 3.72 \\
513 & 3.52 \\
533 & 3.32 \\
\hline
\end{tabular}

As the active area of one adsorbed molecule $\sigma$ is independent of the temperature [13] the mean residence times of the adsorbed molecules can be calculated using Equation (11). In compliance with the theory of Jovanovic we took a value of $0.424 \mathrm{~nm}^{2}$ for $\sigma$, being the effective area of one admolecule multiplied by two. The calculated values of $\tau_{L}$ as a function of the temperature are shown in Table 3.

Based on Equation (12), the heat of adsorption can be calculated for the multilayer range $[13,14,18]$ :

$$
\tau_{L}=\tau_{(o) L} \exp \left(\frac{-Q_{L}}{R T}\right) .
$$

Substituting Equation (12) into Equation (11) and plotting $b^{\prime} \sqrt{T}$ vs. $1 / T$ in a semi-logarithmic plot, the value

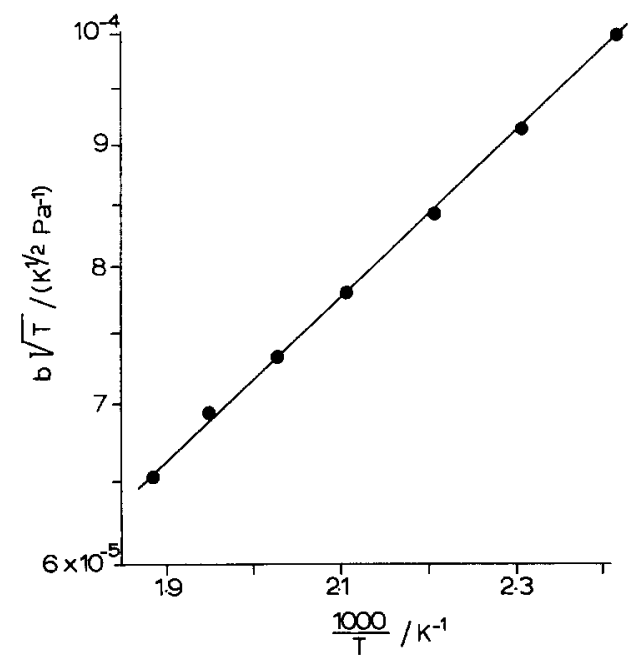

Fig. 5. The temperature dependence of $\sigma \tau_{L} / \sqrt{2 \pi m k}$ in Equation (11) of $Q_{L} / R$ is obtained from the slope of the straight line, provided $Q_{L}$ is independent of the temperature. The result is shown in Figure 5. It can be seen that $Q_{L}$ is indeed independent of temperature. After linear regression we found a value of $-6430 \mathrm{~J} / \mathrm{mol}$ for $Q_{L}$. This is equal to the heat of condensation of methanol at $237.7^{\circ} \mathrm{C}$, just below the critical temperature [19]. Further, from the linear regression we derive a value of $7.76 \cdot 10^{-11} \mathrm{~s}$ for $\tau_{(o) L}$. This is a very large value when compared to the value of $10^{-13} \mathrm{~s}$ as suggested by de Boer [18] for this parameter.

A quantitative interpretation of our adsorption isotherms in the high methanol pressure range (on the right of the line $B B^{\prime}$ in Fig. 3) is much more difficult. Their stepwise form is analogous to the type IV and VIII isotherms in the classification of Jovanovic. However, he explains the occurrence of the irregular steps by capillary condensation phenomena. As already mentioned above, the pressure and temperature range of our experiments is far removed from the region of methanol condensation and capillary condensation is out of the question. In our opinion, the existence of the two "retortion points" on every isotherm - which agree with the points of intersection of the isotherms with the lines $B B^{\prime}$ and $C C^{\prime}$ in Figure $3-$ is possibly related to the pore diameter distribution and surface area distribution of the adsorbent as given in Table 1.

A peculiarity can be observed in the shape of the isotherms in Figure 3: the ratio of the adsorption capacity at the "retortion point" to the calculated monolayer capacity is nearly equal for every isotherm. For the first "retortion points" - corresponding to the line $B B^{\prime}-$ this ratio approximately amounts to 5.5 to 5.9 while for the higher "retortion points" - line $C C^{\prime}$ - it lies between 7.3 and 7.6.

The decrease in the slope of the isotherms in the higher region is possibly caused by the fact that at sufficiently high adsorbate pressures the pores with diameters smaller than a certain value are completely filled with the adsorbate. For cylindrical pores, the number of adsorbed molecules in each succeeding layer will decrease, while for crevice pores it will remain constant. We do not know which pore shapes actually occur in our solids. Another difficulty is to ascribe a value to the thickness of one adsorbed layer. Moreover, we do not know whether the layer thickness will remain the same for each subsequent layer. Due to these unknown factors a quantitative description of the isotherms for the higher adsorbate pressures is impossible. 


\section{Conclusions}

The Peng-Robinson equation of state was found to give an accurate representation of the experimental data for methanol, at reduced pressures of up to 0.15 and in the reduced temperature range of 0.8 to 1.05 . Based on the recent literature data a value of 0.564 was found for the acentric factor of methanol, as distinct from the value of 0.556 which is commonly reported in the literature.

From the experimental results obtained it can be concluded that multilayer adsorption is possible at temperature above the boiling point, even beyond the critical temperatures of the adsorbate. The Jovanovic multilayer adsorption model was found to be valid in this temperature range. The heat of multilayer adsorption calculated from his model was found to be temperature independent. We have not found any experimental adsorption data obtained at conditions comparable to those of our experiments in the literature, so we have no support from other experiments for this rather surprising result.

\section{Acknowledgement}

The authors thank the Twente University of Technology and the Royal Dutch Shell group for the financial support.

\section{References}

1. Peng DYu, Robinson DB (1976) Ind Eng Chem Fundam 15(1):59

2. HirschfelderJO, Curtiss CF, Bird RB (1966) Molecular Theory of Gases and Liquids, 3rd ed, J Wiley \& Sons, New York
3. Bhattacharyya D, Thodos G (1964) J Chem Eng Data 9:530

4. Ambrose D, Sprake CHS, Townsend R (1975) J Chem Ther$\bmod 7: 185$

5. Susic MV, Vucelic DP, Pausak SV, Karaulic DB, MilakovicVucelic V (1969) J Phys Chem 73:1975

6. Hüttig GF (1948) Monatsh Chemie 78:177

7. Brunauer S, Emmett P, Teller E (1938) J Am Chem Soc 60:309

8. Anderson RB (1946) J Am Chem Soc 68:686

9. Smith RN, Pierce C (1948) J Phys Chem 52:1115

10. Steele WA (1956) J Phys Chem 25:819

11. Pickett G (1945) J Am Chem Soc 67:1958

12. Lunde PJ, Kester FL (1975) Chem Eng Sci 30:1497

13. Jovanovic DS (1969) Kolloid ZZ Polym 235:1203

14. Jovanovic DS (1969) Kolloid ZZ Polym 235:1214

15. Yaronets YA, Yaronets M (1980) Bull Acad Sci USSR Div of Chem Sci 29:870

16. Jaroniec M, Sokolowski S, Waksmudzki A (1976) Roczn Chemii 50:779

17. Sokolowski S, Jaroniec M, Waksmudzki A (1976) Roczn Chemii 50:1149

18. de Boer JH (1968) The Dynamical Character of Adsorption, Oxford University Press, London

19. Radosz M, Lydersen A (1980) Chem Ing Tech 52:756

Received August 27, 1985; accepted September 23, 1985

Authors' address:

M. Kuczynski

Chemical Reaction Engineering Laboratories

Department of Chemical Engineering

Twente University of Technology

P.O.Box 217

7500 AE Enschede, The Netherlands

Correspondence should be directed to K. R. Westerterp, same address. 